\title{
Proton Pump Inhibitors: Their Misuse, Overuse, And Abuse
}

\author{
${ }^{1,}$ Ashton M. Atkins, ${ }^{2,}$ M. Chandra Sekar ${ }^{*}$ R.Ph., Ph.D \\ ${ }^{1,2,}$ The University of Findlay College of Pharmacy 1000 North Main Street \\ Findlay, Ohio 45840
}

\begin{abstract}
Proton pump inhibitors (PPI) are a relatively new class of potent gastric acid-suppressing agents that are used in the treatment of multiple gastrointestinal (GI) disorders. As with any medication it is important that PPIs should only be prescribed when appropriate, and mainly for FDA-approved indications. The improper use of proton pump inhibitors can lead to unwanted adverse effects and increase the chances for drug induced disease, such as gastric cancer. There is some recent evidence indicating that improper use of PPI is rising. Findings from our literature review and clearly suggest that PPI's are being abused through overuse, and the various unintentional consequences of their overuse; not only to the individuals taking the medications, but also the additional cost incurred by the healthcare system.
\end{abstract}

Keywords-abuse, GERD, overuse, proton pump inhibitor, use

\section{INTRODUCTION}

Proton pump inhibitors (PPI) are a relatively new class of potent gastric acid-suppressing agents that are used in the treatment of multiple gastrointestinal (GI) disorders (Table 1). Proton pump inhibitors exert their effect by inhibiting the $\mathrm{H}^{+} / \mathrm{K}^{+}$-adenosine triphosphatase (ATPase), or proton pump, which is located in the highly acidic lumen of parietal cells. ${ }^{[1]}$ This highly acidic environment enables the PPI to become protonated to its active metabolite which then irreversibly inhibits the activity of the proton pump, resulting in an increase of gastric $\mathrm{pH}$. As opposed to $\mathrm{H}_{2}$-receptor antagonists, another class of GI agents, proton pump inhibitors are longer-acting and produce a greater degree of gastric acid suppression, thus making them more effective. Proton pump inhibitors also have a utility in the prophylaxis of stress- and NSAID-induced peptic ulcer disease (PUD). Clinicians are more inclined to prescribe a PPI rather than an alternative pharmaceutical agent for the treatment of GI disorder because of their higher efficacy and because of the wide range of conditions treated by these agents (Table 1). The use of these agents has increased by $456 \%{ }^{[2]}$ since the 1990 's. As a result, these drugs have become one of the world's most frequently prescribed medications. Several clinical trials have demonstrated that proton pump inhibitors are both safe and well tolerated: when used appropriately. However PPI's overuse and accompanying misuse increases the likelihood of adverse effects. Because most gastric-acid related disorders require long-term treatment, the risk for clinically significant adverse drug interactions is probable in patients who are receiving other medications in conjunction with a PPI. It is imperative that clinicians prescribe PPIs only when proven necessary.

\section{Non-Compliant PPI Prescriptions}

Several reports have suggested that PPIs are being overused in hospital and ambulatory care settings. ${ }^{[2,3]}$ The appropriateness of PPI prescriptions in some hospitals is as low as $19 \%{ }^{[2]}$ Multiple variables should be taken into account before prescribing a proton pump inhibitor including: (i) dosages, duration of therapy and clinical reasons for the use of a PPI accompanied by an assessment of the appropriateness of the treatment; (ii) whether the use of $\mathrm{H}_{2}$-receptor antagonists is appropriate before commencing with a proton pump inhibitor, and (iii) how often patients receiving a proton pump inhibitor for gastro-esophageal reflux disease (GERD) were also being treated with other medications which are known to aggravate or cause GERD. ${ }^{[3]}$ With these factors in mind, several clinical studies performed in the hospital setting often conclude that the percentage of patients admitted to a hospital for gastrointestinal disorders already taking a PPI is substantially less than the percentage of those who were prescribed a PPI upon discharge from the hospital. ${ }^{[2,3,4,5]} \mathrm{A}$ crosssectional, prescription-indication drug-utilization study conducted in Spain concluded that out of 328 patients in a single hospital $28.65 \%$ were prescribed a PPI at admission, $82.62 \%$ were prescribed a PPI during their stay, and $54.75 \%$ at discharge, of which improper indications for PPI prescription were $74.47 \%, 61.25 \%$, and $80.24 \%$ respectively. ${ }^{[5]}$ Other similar studies have also concluded that of those patients who were prescribed a proton pump inhibitor, either during their stay at the hospital or upon discharge, a significantly large percentage were guideline non-compliant. ${ }^{[2,3,4,5,6,7]}$ 
A recent study performed in the state of Maryland incorporated two different hospitalist groups: academic and non-academic hospitals. All PPI prescriptions dispensed at the hospitals were investigated in accordance to FDA guideline compliance. ${ }^{[2]}$ As shown in Figure 1, GI prophylaxis for low risk patients was the leading indication accounting for $82 \%$ of all non-compliant proton pump inhibitor prescriptions. ${ }^{[2]}$ Improper prescription of proton pump inhibitors can have a very negative effect on those taking the medications, especially if a follow-up examination is not performed. Recently, a combination product containing both Naproxen and esomeprazole was launched in the United States. The justification for this is that if a medication contains both a PPI and an NSAID, the PPI will counteract any gastrointestinal adverse effects caused by the NSAID. While this may seem like a good idea, combination products will only increase the chances of the general population being overexposed to proton pump inhibitors. As stated earlier, a proton pump inhibitor should only be used when all other acid suppressing agents have failed. The adverse effects sustained after a long duration of treatment with PPIs, or the use of a PPI with a variety of other medications can be debilitating, and in some cases even lethal, if left untreated.

\section{Adverse Effects}

Most medications have an extensive list of non-severe or moderate adverse effects. These adverse effects, which will differ in magnitude according to individual sensitivity, include headache, dizziness, diarrhea, fatigue, abdominal pain, nausea, dry mouth, etc. Although many individuals may not experience such effects, however, they are not uncommon. Therefore, responsible drug use is imperative when it comes to the prevention of adverse effects. Because PPI decrease the acidity of the stomach, the main concern from the effect of profound acid suppression is hypergastrinaemia. Patients receiving a PPI are also susceptible to the colonization of ingested pathogens which can lead to bacterial gastroenteritis. ${ }^{[1,3]}$ The US Food and Drug Administration (FDA) just recently announced that overexposure or prolonged use of a proton pump inhibitor may be associated with a higher risk of infection by the deadly bacteria Clostridium Difficile (CDAD). ${ }^{[8]}$ Evidence shows there is a distinct link between prolonged gastric acid suppression, hypergastrinaemia and neuroendocrine cell hyperplasia, which may allow the production of carcinogenic substances. ${ }^{[3]}$ Often times symptoms that are linked to the development of gastric cancer are masked from endoscopy. It is for this reason that plasma gastrin levels should be monitored in those individuals where long-term treatment with a proton pump inhibitor is recommended.

Due to the fact that proton pump inhibitors are commonly prescribed to regulate and prevent symptoms of a chronic implacable condition, it is probable that the duration of therapy may exceed more than four years. This prolonged treatment is believed to hinder calcium absorption in the small intestine. ${ }^{[9]}$ The ability of the small intestine to absorb calcium salts is highly $\mathrm{pH}$ dependent, and since proton pump inhibitors cause an increase in gastric $\mathrm{pH}$, calcium salts are rendered insoluble and cannot be absorbed. This inhibition of calcium absorption has a direct correlation to osteoporotic fractures in those individuals taking a PPI. A study conducted in Canada determined that after seven years of continuous exposure to a PPI, there was a statistically significant increase in osteoporosis-related fractures, and an increase risk of hip fracture after five years. ${ }^{[9]}$ As bone mineralization and resorption takes many years, and because of the subtle effect that proton pump inhibitors have on bone mineralization, several years may be required before it has a measurable clinical outcome.

\section{Pharmacokinetic Drug Interactions}

As the number of medications that an individual takes increases, so does the propensity for drug interactions. Also to be noted is that gastric acid-related conditions and polypharmacy in the elderly are not uncommon. The main mechanisms through which PPIs generally cause an unwanted drug interaction is by increasing gastric $\mathrm{pH}$ and inhibiting intestinal first-pass metabolism and hepatic clearance of other drugs. ${ }^{[1]}$ Ketocanazole is a prime example of a drug with pharmacokinetics that are affected by changes in gastric $\mathrm{pH} .{ }^{[1]}$ This interaction reduces the antifungal activity of ketoconazole, decreasing the therapeutic potency of the drug. Several clinical studies have determined the significant drug interactions with available proton pump inhibitors in the United States

\subsection{Omeprazole}

Studies have shown that omeprazole reduces the clearance of diazepam by $20-26 \%$, and competitively inhibits phenytoin and warfarin. There have been case studies of an omeprazole-induced detrimental increase in the anticoagulant effect of warfarin and phenprocoumon after concomitant use of omeprazole. ${ }^{[1]}$

\subsection{Esomeprazole}

The pharmacokinetic drug interactions of omeprazole and esomeprazole are very similar because they are both subject to the same metabolic transformations. The effect of esomeprazole on diazepam has the likelihood to disrupt motor coordination and vigilance of individuals receiving both drugs at the same time. 
Esomeprazole also has an effect on the clearance of warfarin from the body. Therefore, patients receiving treatment with esomeprazole, or any proton pump inhibitor, should be monitored for increase in INR (international normalized ratio) and prothrombin time..$^{[1]}$

\subsection{Pantoprazole}

Clinical studies in healthy individuals have shown that there are no significant drug interactions when pantoprazole was used in combination with any of these medications listed in table 2 . Being that pantoprazole has such a low potential for interaction with any other medications this would be a suitable proton pump inhibitor for a patient who needs to be on PPI therapy for an extended amount of time, or for an individual taking multiple medications with narrow therapeutic windows.

\subsection{Lansoprazole}

Certain in vitro studies have concluded that lansoprazole can competitively inhibit the metabolism of some medications just as omeprazole, but the drug interactions are less significant. ${ }^{[1]}$ In order to obtain a more detailed pharmacokinetic drug interaction profile for lansoprazole, more extensive clinical research needs to be done.

\subsection{Rabeprazole}

Due to lack of research, it is not well known whether or not there are any clinically significant drug interactions induced by rabeprazole. However, it is known that rabeprazole, as well as all proton pump inhibitors, increase gastric $\mathrm{pH}$ which has an effect on the bioavailability of both digoxin and ketoconazole. ${ }^{[1]}$ In general, lansoprazole, pantoprazole, and rabeprazole appear to have a lesser potential to induce any significant drug interactions as opposed to omeprazole and esomeprazole. In practice there is little difference in efficacy among the different proton pump inhibitors at similar doses, and this may become an important deciding factor for a clinician when prescribing a PPI. Because all proton pump inhibitors are equally effective at appropriate recommended doses, their proclivity to bring about a significant drug interaction should be taken into consideration. This becomes particularly important in elderly patients in which most GI disorders are common and they are often taking multiple medications at the same time.

\section{Economic Effect Of PPI Overuse}

Very often proton pump inhibitors are prescribed and overused without documented valid indications. This inappropriate use comes with a cost that rapidly accumulates over time. If the rate at which non-FDA compliant prescriptions were being dispensed could be controlled, this would result in an immense cost savings to both the government and the general public. To put into perspective the magnitude of the economic effect that the overuse of proton pump inhibitors has, a retrospective cohort cost study was performed in Michigan in a specific ambulatory care setting. Those involved in the study found that the total cost in a year of inappropriate PPI use, whether it be non-compliant indication or inappropriate documentation, was $\$ 233,994^{[10]}$ based on over-the-counter costs and $\$ 1,566,252^{[10]}$ based on average whole sale price costs. Another clinical study geared toward the cost impact of the overuse of proton pump inhibitors in non-ICU patients found that an annual cost savings of over $\$ 35,0000^{[6]}$ could be possible if precautions were set in place to stop the non-compliant prescribing of PPIs.

\section{Conclusion}

In conclusion, it is clear that PPIs are currently being both overused and misused. While all the publications cited are from studies conducted in United States or other European countries, conclusion made in this article are widely applicable, as in developing country like India, where over five hundred branded formulations of PPI are available, probability of misuse and abuse increases exponentially. Although a safe and very effective class of pharmaceutical agent, proton pump inhibitors should be used only when there is documented evidence of a GI disorder that cannot be treated with an $\mathrm{H}_{2}$-receptor antagonist, and where a PPI use is clinically justified. Increased Clinician awareness on appropriate PPI prescription will lead to better patient outcome at lower cost.

\section{References}

[1] H Blume, F. Donath, A. Warnke, B. Schug, Pharmacokinetic drug interaction profiles of proton pump inhibitors, Drug Safety, 29, 2006, 769-780.

[2] S Eid, A. Boueiz, S. Paranji, C. Mativo, R Landis, M. Abougergi, Patterns and predictors of proton pump inhibitor overuse among academic and non-academic hospitalists. Internal Medicine, 49, 2010, 2561-2568.

[3] M Naunton, G. Peterson, M. Bleasel, Overuse of proton pump inhibitors, Journal of Clinical Pharmacy and Therapeutics, 25, 2000, 333-338.

[4] L Pasina, A. Nobili, M. Tettamanti, F. Salerno, S. Corrao, A. Marengoni, et al. Prevalence and appropriateness of drug prescriptions for peptic ulcer and gastroesophageal reflux disease in a cohort of hospitalized elderly, Am J. Managed Care,16, 2010, 228-234 
[5] E Ramirez, S. Lei, A. Borobia, E. Pinana, S. Fudio, R. Munoz et al, Overuse of PPIs in patients at admission, during treatment, and at discharge in a tertiary Spanish hospital, Current Clinical Pharmacology, 5, 2010, 288-297

[6] S Nasser, J. Nassif, H. Dimassi, Clinical and cost impact of intravenous proton pump inhibitor use in non-ICU patients, World J Gastroenterol, 16, 2010, 982-986.

[7] A Lanas, J. Esplugues, J. Zapardiel, E. Sobreviela, Education-based approach to addressing non-evidence-based practice in preventing NSAID-associated gastrointestinal complications, World J Gastroenterol, 15, 2009 5953-5959.

[8] R Lowes, Proton pump inhibitors linked to c difficile diarrhea. Medscape. 2012.

[9] L Targownik, L. Lix, C. Metge, H. Prior, S. Leung, W. Leslie, Use of proton pump inhibitors and risk of osteoporosis-related fractures, CMAJ, 179, 2008, 319-326

[10] J Heidelbaugh, K. Goldberg, J. Inadomi, Magnitude and economic effect of overuse of antisecretory therapy in the ambulatory care setting, Current Clinical Pharmacology, 5, 2010, 288-297.

Table 1 - Generic PPI along with their FDA approved indications

\begin{tabular}{|c|c|c|c|c|c|c|}
\hline FDA approved indications & 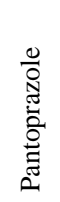 & 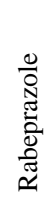 & 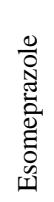 & 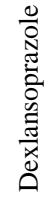 & 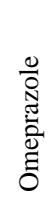 & 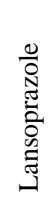 \\
\hline Healing of erosive esophagitis & $\mathrm{X}$ & $\mathrm{X}$ & $\mathrm{X}$ & $\mathrm{X}$ & $\mathrm{X}$ & $\mathrm{X}$ \\
\hline Maintenance of healing of erosive esophagitis & $\mathrm{X}$ & $\mathrm{X}$ & $\mathrm{X}$ & $\mathrm{X}$ & $\mathrm{X}$ & $\mathrm{X}$ \\
\hline Symptomatic gastroesophageal reflux disease & $\mathrm{X}$ & $\mathrm{X}$ & $\mathrm{X}$ & $\mathrm{X}$ & $\mathrm{X}$ & $\mathrm{X}$ \\
\hline $\begin{array}{l}\text { H. pylori eradication in combination with } \\
\text { antibiotics }\end{array}$ & & $\mathrm{X}$ & $\mathrm{X}$ & & $\mathrm{X}$ & $\mathrm{X}$ \\
\hline Short-term treatment of active gastric ulcer & & & & & $\mathrm{X}$ & $\mathrm{X}$ \\
\hline Short-term treatment of active duodenal ulcer & & $\mathrm{X}$ & & & $\mathrm{X}$ & $\mathrm{X}$ \\
\hline Maintenance of healed duodenal ulcer & & & & & & $\mathrm{X}$ \\
\hline Healing of NSAID-associated gastric ulcer & & & & & & $\mathrm{X}$ \\
\hline Risk reduction of NSAID-associated gastric ulcer & & & $\mathrm{X}$ & & & $\mathrm{X}$ \\
\hline $\begin{array}{l}\text { Risk reduction of upper gastrointestinal bleeding } \\
\text { in critically ill patients }\end{array}$ & & & & & $\mathrm{X}$ & \\
\hline $\begin{array}{l}\text { Pathological hypersecretory conditions incl. } \\
\text { Zollinger-Ellison Syndrome }\end{array}$ & $\mathrm{X}$ & $\mathrm{X}$ & $\mathrm{X}$ & & $\mathrm{X}$ & $\mathrm{X}$ \\
\hline $\begin{array}{l}\text { Symptomatic gastroesophageal reflux } \\
\text { disease/erosive esophagitis in children }\end{array}$ & & $X$ & $\mathrm{X}$ & & $\mathrm{X}$ & $X$ \\
\hline
\end{tabular}




\section{Compliant \\ Prescriptions}

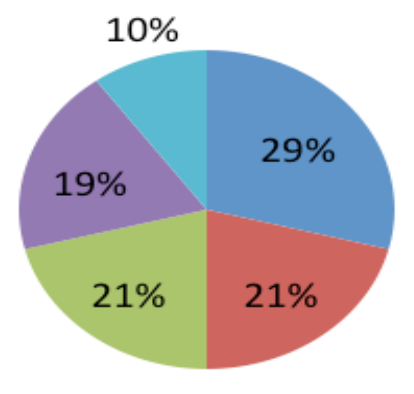

Dyspepsia

- Gastritis/

Dueodenitis/

Ulcer

GERD by

history of EGD

\section{Non-Compliant Prescriptions}

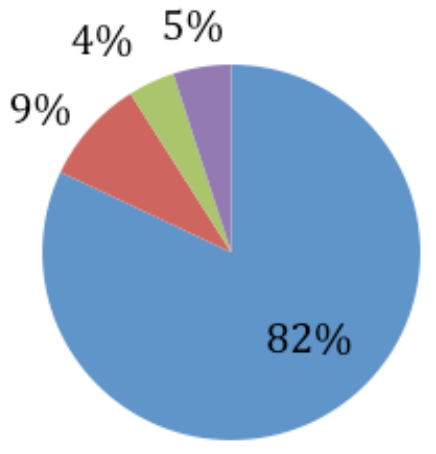

GI

Prophylaxis

Abd pain and nausea

Non cardiac chest pain

ather

Figure 1: indications for guidelines compliant and non-compliant proton pump inhibitor prescriptions ${ }^{[2]}$ 